\title{
Tests of CPT symmetry and quantum coherence with entangled neutral kaons at KLOE
}

\section{R. D'Amico ${ }^{a, b, *}$ on behalf of the KLOE-2 Collaboration}

${ }^{a}$ I.N.F.N. Sezione di Roma,

P.le A. Moro, 2, I-00185 Rome, Italy

${ }^{b}$ Physics Department, La Sapienza University of Rome,

P.le A. Moro, 5, Rome, Italy

E-mail: riccardo.damico@roma1.infn.it

\begin{abstract}
The entanglement in the neutral kaon pairs observed by the KLOE experiment at the DAФNE $\phi$-factory is a unique tool to test discrete symmetries and quantum coherence at the utmost sensitivity, in particular strongly motivating the experimental searches of possible CPT violating effects, which would unambiguously signal New Physics.

We will present the final result of the measurement of the decoherence and CPT violation parameters in $K_{S} K_{L} \rightarrow \pi^{+} \pi^{-} \pi^{+} \pi^{-}$with an improved statistical accuracy of a factor $\sim 2$ with respect to literature.
\end{abstract}

*** The European Physical Society Conference on High Energy Physics (EPS-HEP2021), ***

*** 26-30 July $2021 * * *$

*** Online conference, jointly organized by Universität Hamburg and the research center DESY ***

${ }^{*}$ Speaker 


\section{Introduction}

Neutral kaon pairs are produced at DAФNE in a coherent quantum state with the $\phi$ meson quantum numbers $J^{C P}=1^{--}$:

$$
|i\rangle=\frac{1}{\sqrt{2}}\left\{\left|K^{0}\right\rangle\left|\bar{K}^{0}\right\rangle-\left|\bar{K}^{0}\right\rangle\left|K^{0}\right\rangle\right\}=\frac{N}{\sqrt{2}}\left\{\left|K_{S}\right\rangle\left|K_{L}\right\rangle-\left|K_{L}\right\rangle\left|K_{S}\right\rangle\right\}
$$

where $N \simeq 1$ is a normalization factor. The CP violating channel $\phi \rightarrow K_{S} K_{L} \rightarrow \pi^{+} \pi^{-} \pi^{+} \pi^{-}$is ideal to observe the quantum interference between the two kaons initially in the entangled state in eq. (1). The measured $\Delta t$ distribution, with $\Delta t$ the absolute value of time difference of the two $\pi^{+} \pi^{-}$ decays, can be fitted with the distribution:

$$
I\left(\pi^{+} \pi^{-}, \pi^{+} \pi^{-} ; \Delta t\right) \propto e^{-\Gamma_{S} \Delta t}+e^{-\Gamma_{L} \Delta t}-2\left(1-\zeta_{S L}\right) e^{-\frac{\Gamma_{S}+\Gamma_{L}}{2} \Delta t} \cos (\Delta m \Delta t)
$$

where the quantum mechanical expression in the $\left\{K_{S} K_{L}\right\}$ basis has been modified, following the Furry's hypothesis [1], with the introduction of a decoherence parameter $\zeta_{S L}$. Analogously, a $\zeta_{0 \overline{0}}$ can be defined in the $\left\{K^{0} \bar{K}^{0}\right\}$ basis [2].

At a microscopic level, in a quantum gravity picture, space-time might be subjected to inherent non-trivial quantum metric and topology fluctuations at the Planck scale $\left(\sim 10^{-33} \mathrm{~cm}\right)$. This spacetime structure might induce a pure state to evolve into a mixed one [3], i.e. decoherence effects that necessarely entail CPT violation [4].

The above mentioned decoherence mechanism can be described by a density matrix formalism with a modifed Liouville-von Neumann equation [5][6]:

$$
\frac{d \rho}{d t}=-i \mathbf{H} \rho+i \rho \mathbf{H}^{\dagger}+L(\rho ; \alpha, \beta, \gamma)
$$

where $\rho$ is the density matrix and the extra term $L(\rho ; \alpha, \beta, \gamma)$ would induce decoherence in the system and depends on three real parameters, $\alpha, \beta$ and $\gamma$ which violate CPT symmetry and quantum mechanics. In the entangled kaon system at KLOE this decoherence model can be tested in the symplifing hypotesis of complete positivity, i.e. $\alpha=\gamma$ and $\beta=0$ with $\gamma$ as the independent parameter describing the phenomenon that has mass dimension and is guessed to be at most of the order of $O\left(m_{K}^{2} / M_{\text {Planck }}\right) \sim 2 \cdot 10^{-20} \mathrm{GeV}$ [7][8].

As discussed above, in a quantum gravity framework inducing decoherence, the CPT operator is ill-defined. The resulting loss of particle-antiparticle identity could induce a breakdown of the correlation of state (1) imposed by Bose statistics. As a result the initial state (1) can be parametrized in general as [9][10]:

$$
\begin{aligned}
|i\rangle= & \frac{1}{\sqrt{2}}\left[\left|K^{0}\right\rangle\left|\bar{K}^{0}\right\rangle-\left|\bar{K}^{0}\right\rangle\left|K^{0}\right\rangle+\omega\left(\left|K^{0}\right\rangle\left|\bar{K}^{0}\right\rangle+\left|\bar{K}^{0}\right\rangle\left|K^{0}\right\rangle\right)\right] \\
& \propto\left[\left|K_{S}\right\rangle\left|K_{L}\right\rangle-\left|K_{L}\right\rangle\left|K_{S}\right\rangle+\omega\left(\left|K_{S}\right\rangle\left|K_{S}\right\rangle-\left|K_{L}\right\rangle\left|K_{L}\right\rangle\right)\right]
\end{aligned}
$$

where $\omega$ is a complex parameter describing a completely novel CPT violation phenomenon, not included in previous analyses. Its order of magnitude could be at most $|\omega| \sim\left[\left(m_{K}^{2} / M_{\text {Planck }}\right) / \Delta \Gamma\right]^{1 / 2} \sim$ $10^{-3}$. Here we present the results on $\zeta_{S L}, \zeta_{0 \overline{0}}, \gamma, \mathfrak{R} \omega, \mathfrak{J} \omega,|\omega|$ and $B R\left(\phi \rightarrow K_{S} K_{S}\right)$ from the analysis of a data sample corresponding to an integrated luminosity $L \approx 1.7 \mathrm{fb}^{-1}$ collected in 2004-05.

\section{Decoherence and CPT Violation Data Analysis}

For the present analysis two decay vertices with only two tracks each are required. For each vertex the same kinematic criteria are used for the sample selection, furthermore since the $\Delta t$ resolution 
is strongly correlated with the opening angle of the pion tracks $\left(\theta_{ \pm}\right)$and deteriorates at large values of $\theta_{ \pm}$a cut to eliminate large opening angles values has been applied rejecting events with $\cos \theta_{ \pm}<-0.975$.

After event selection, the sources of irreducible background are the regeneration in the thin $(50 \mu \mathrm{m})$ $B e$ beam pipe near the IP and the direct non resonant $e^{+} e^{-} \rightarrow \pi^{+} \pi^{-} \pi^{+} \pi^{-}$process. The latter after event selection is relatively small but concentrated in the most sensitive region for decoherence, therefore needs to be carefully evaluated. To study this background the 2-dimensional invariant mass distribution of the vertices was observed and an unbinned maximum likelihood fit was performed in order to evaluate the number of background events that is found to be, in the nominal cut region: $39 \pm 5$ for $\Delta t<1 \tau_{S}, 6 \pm 2$ for $1 \tau_{S}<\Delta t<2 \tau_{S}$ and $6 \pm 2$ for $2 \tau_{S}<\Delta t<3 \tau_{S}$.

The experimental data distributions have been fitted with all the models described in section (1) taking into account backgrounds, efficiencies and $\Delta t$ resolution. Both data distribution and theory for the $\zeta_{S L}$ model are shown as an example in figure (1a). For the $\omega$ model it was also produced the contour plot, shown in figure (1b), for the $68 \%$ and $95 \%$ of confidence level. The systematic uncertainties are shown in table (1).

The final results of this analysis for all described models are:

$$
\begin{aligned}
& \zeta_{S L}=\left(0.1 \pm 1.6_{\text {stat }} \pm 0.7_{\text {syst }}\right) \cdot 10^{-2} \\
& \zeta_{0 \overline{0}}=\left(-0.05 \pm 0.80_{\text {stat }} \pm 0.37_{\text {syst }}\right) \cdot 10^{-6} \\
& \gamma=\left(0.13 \pm 0.94_{\text {stat }} \pm 0.42_{\text {syst }}\right) \cdot 10^{-21} \mathrm{GeV} \\
& \mathfrak{R} \omega=\left(-2.3_{-1.5}^{+1.9}{ }_{\text {stat }} \pm 0.6_{\text {syst }}\right) \cdot 10^{-4} \\
& \mathfrak{J} \omega=\left(-4.1_{-2.6 \text { stat }}^{+2.8} \pm 0.9_{\text {syst }}\right) \cdot 10^{-4} \\
& |\omega|=\left(4.7 \pm 2.9_{\text {stat }} \pm 1.0_{\text {syst }}\right) \cdot 10^{-4}
\end{aligned}
$$

The results on $\zeta_{S L}, \zeta_{0 \overline{0}}$ and $\gamma$ parameters, that are constrained to be positive, can be translated into upper limits:

$$
\begin{aligned}
& \zeta_{S L}<0.030(90 \% \text { C.L. }) \\
& \zeta_{0 \overline{0}}<1.4 \cdot 10^{-6}(90 \% \text { C.L. }) \\
& \gamma<1.8 \cdot 10^{-21} \mathrm{GeV}(90 \% \text { C.L. })
\end{aligned}
$$

while from the value of $|\omega|$, since $|\omega|^{2}=\frac{B R\left(\phi \rightarrow K_{S} K_{S}\right)}{B R\left(\phi \rightarrow K_{S} K_{L}\right)}$ from the modified initial state (4), it can be derived an upper limit for the branching ratio of the $\phi \rightarrow K_{S} K_{S}$ decay:

$$
B R\left(\phi \rightarrow K_{S} K_{S}\right)<2.4 \cdot 10^{-7}(90 \% \text { C.L. })
$$

Although the results are consistent with no deocherence effects and no CPT violation, the statistical uncertainties have been reduced by half with respect to previous KLOE measurements [11] and represent one of the most precise tests of quantum coherence in an entangled neutral system. 

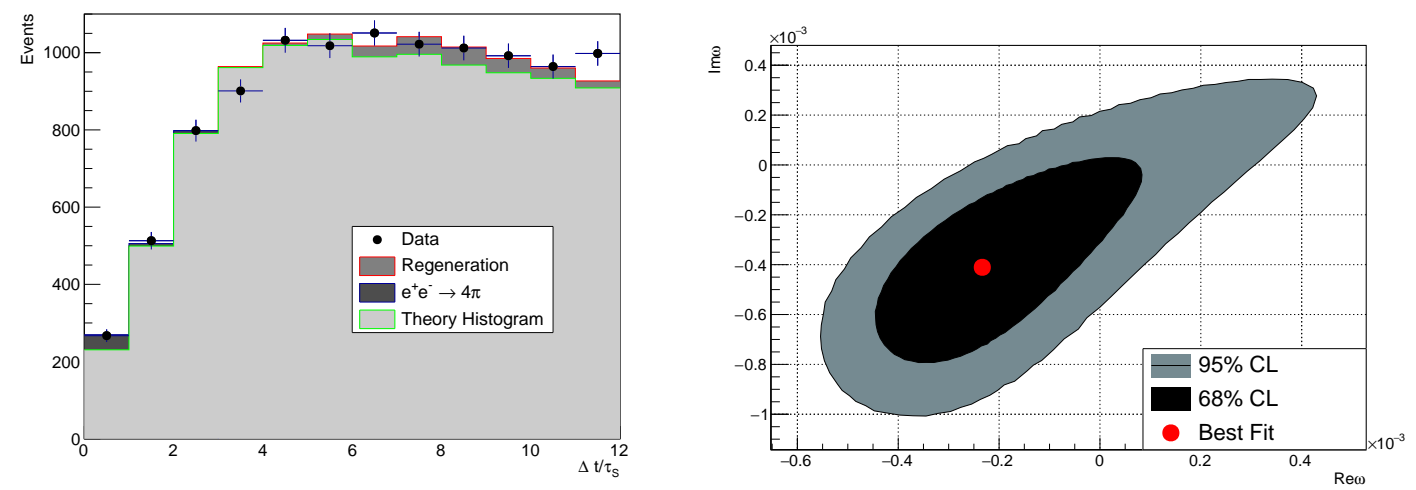

(a) Data and theoretical distribution from the fit for (b) Contour plot of $\mathfrak{J} \omega$ vs $\mathfrak{R} \omega$ for the confidence level $68 \%$ the $\zeta_{S L}$ decoherence model in which are displayed all and $95 \%$ different background contributions.

Figure 1

\begin{tabular}{|c|c|c|c|c|c|c|}
\hline & $\delta \zeta_{S L} \cdot 10^{2}$ & $\delta \zeta_{0 \overline{0}} \cdot 10^{7}$ & $\delta \gamma \cdot 10^{21} \mathrm{GeV}$ & $\delta \mathfrak{R} \omega \cdot 10^{4}$ & $\delta \mathfrak{J} \omega \cdot 10^{4}$ & $\delta|\omega| \cdot 10^{4}$ \\
\hline Cut stability & \pm 0.56 & \pm 2.9 & \pm 0.33 & \pm 0.53 & \pm 0.65 & \pm 0.78 \\
$4 \pi$ Background & \pm 0.37 & \pm 1.9 & \pm 0.22 & \pm 0.32 & \pm 0.19 & \pm 0.32 \\
Regeneration & \pm 0.17 & \pm 0.9 & \pm 0.10 & \pm 0.06 & \pm 0.63 & \pm 0.58 \\
Resolution & \pm 0.18 & \pm 0.9 & \pm 0.10 & \pm 0.15 & \pm 0.09 & \pm 0.15 \\
Input & \pm 0.04 & \pm 0.2 & \pm 0.02 & \pm 0.03 & \pm 0.09 & \pm 0.07 \\
\hline Total & \pm 0.71 & \pm 3.7 & \pm 0.42 & \pm 0.64 & \pm 0.93 & \pm 1.04 \\
\hline
\end{tabular}

Table 1: Systematic uncertainties on all decoherence parameters, for the cut stability, the regeneration, the resolution, the $4 \pi$ background and the physical constants.

\section{References}

[1] W. H. Furry, Note on the quantum-mechanical theory of measurement, Phys.Rev., vol. 49, pp. 393-399, 1936.

[2] R. A. Bertlmann, W. Grimus, and B. C. Hiesmayr, Quantum mechanics, Furry's hypothesis, and a measure of decoherence in the $K^{0} \bar{K}^{0}$ system, Phys. Rev. D, vol. $60114032,1999$.

[3] S. W. Hawking, "The unpredictability of quantum gravity", Comm. Math. Phys., vol. 87, no. 3, pp. 395-415, 1982.

[4] R.Wald, Quantum gravity and time reversibility, Phys. Rev. D, vol. 21, no.10, 1980.

[5] Ellis, J.; Hagelin, J.S.; Nanopoulos, D.V.; Srednicki, M. Search for violations of quantum mechanics, Nucl. Phys. B vol. 241, 381-405, 1984.

[6] Ellis, J.; Lopez, J.L.; Mavromatos, N.E.; Nanopoulos, D.V., Precision tests of CPT symmetry and quantum mechanics in the neutral kaon system, Phys. Rev. D vol. 53, 3846-3870, 1996 [hep-ph/9505340].

[7] F. Benatti, R. Floreanini, Complete positivity and correlated neutral kaons, Phys. Lett. B vol .468, pp. 287-293, 1999 [hep-ph/9910508].

[8] F. Benatti, R. Floreanini, Completely positive dynamics of correlated neutral kaons, Nucl. Phys. B vol. 511, pp. 550-576, 1998.

[9] J. Bernabéu, N. Mavromatos, J. Papavassiliou, Novel Type of CPT Violation for Correlated EinsteinPodolsky-Rosen States of Neutral Mesons., Phys. Rev. Lett., vol. 92, 131601, 2004.

[10] J. Bernabéu, N.E. Mavromatos, J. Papavassiliou, A. Waldron-Lauda, Intrinsic CPT violation and decoherence for entangled neutral mesons., Nucl. Phys. B, vol. 744, 180-206, 2006.

[11] KLOE collab., First observation of quantum interference in the process $\phi \rightarrow K_{S} K_{L} \rightarrow \pi^{+} \pi^{-} \pi^{+} \pi^{-}: A$ test of quantum mechanics and CPT symmetry, Phys.Lett B vol 642, 315-321, 2006 [hep-ex/0607027]. 